\title{
Costo y desenlace de la infección de artroplastía de cadera. Estudio de caso y control
}

\author{
Osvaldo Iribarren B., Alejandra Álvarez C., Cristian Rodríguez C. \\ Mónica Ferrada M., Hugo Hernández V. y Lilian Dorn H.
}

\section{Cost and outcome of hip's arthroplasty nosocomial infection. Case and control study}

Case-control study for evaluation of cost and outcome of nosocomial surgical site infection in primary hip arthroplasty during a period of 5 years. Mean hospitalization time in the orthopedic service was 54 days for cases, and 13 days for control $(\mathrm{p}<0.05)$. Mean hospitalization time in Intensive Care Unit (ICU) was 1.1 days for cases. There were 0.83 post primary surgery interventions in cases, and a mean of 2.08 bacteriological cultures in each case. Controls didn't have hospitalizations in the ICU surgical reinterventions nor cultures necesary. The mean cost of infected patients was US \$: 6,174.8. Mean cost in controls was US \$: 2,354.7. The excess of cost due to infections was US $\$: 2,354.7$ on the average. Outcomes in cases were: normal function $30.8 \%$; moderate or serious sequelea $46.2 \%$; death rate $15,4 \%$. The measured parameters contributed to raise case costs, and they caused an unsatisfactory outcome for two third of the patients.

Key words: Nosocomial infection, hip arthroplasty, outcome of arthroplasty infection, nosocomial infection costs.

Palabras claves: Infección intrahospitalaria, artroplastía de cadera, desenlace en infección de artroplastía, costo de infección intrahospitalaria.

\section{Introducción}

$\mathrm{L}$ a artroplastía total de cadera ha resistido la prueba del tiempo como una operación que elimina el dolor y mejora la calidad de vida de muchos pacientes con enfermedad degenerativa de la cadera. El concepto de reemplazo de la cadera fue introducido por Wiles en 1938, quien fijó una esfera de acero inoxidable mediante un vástago, al cuello femoral y un capuchón de acero inoxidable fijado a la pelvis, mediante placas y tornillos de igual material ${ }^{1}$.

En 1961, Sir John Charnley, desarrolló una forma de fijar el implante protésico utilizando un cemento acrílico autofraguable, que ha demostrado éxito en su estabilidad en evaluaciones a largo plazo -más de 15 años- en tanto otros implantes cementados, han tenido resultados similares a 10 años de seguimiento ${ }^{2,3}$.

La osteartrosis primaria es la causa más frecuente de cirugía reconstructiva de la cadera. Otras patologías que requieren reemplazo protésico, son osteoartrosis secundarias a luxación congénita, mesenquimopatías, tumores, necrosis avascular de la cabeza femoral o fracturas. La estimación de la incidencia de esta cirugía se ha establecido en 2,23 por cada 1.000 personas sobre 50 años de edad, excluida la cirugía originada por fractura de cadera ${ }^{4}$.

La infección de prótesis articulares tiene una incidencia relativamente baja, que fluctúa entre 2 y $5 \%{ }^{5,6}$, pero que tiene consecuencias clínicas devastadoras por sus implicancias en el uso de terapias antimicrobianas prolongadas, en la aplicación de aseos quirúrgicos frecuentes, en el retiro de material protésico infectado y en las secuelas temporales y definitivas que generan en los pacientes. El aumento de los costos sobre los inicialmente programados y la necesidad de una estadía prolongada, son una realidad obligada en estas condiciones.

El primer objetivo del presente estudio es informar sobre el exceso de costo atribuible a la infección de herida operatoria en artroplastía de cadera, valorando los costos monetarios directos de la atención. El segundo objetivo de este estudio es conocer el desenlace de los pacientes portadores de infección de artroplastía de cadera.
Universidad Católica del Norte Coquimbo, Chile Escuela de Medicina (OIB, HHV) Estudiante de Medicina (AAC, CRC) Hospital San Pablo de Coquimbo, Chile

Comité de Control de Infecciones Intrahospitalarias (OIB, MFM, LDH)

Recibido: 28 diciembre 2005 Aceptado: 8 agosto 2006

Correspondencia a: Osvaldo Iribarren Brown oiribarren@123.cl osvaldoi@ucn.cl 


\section{Pacientes y Métodos}

El presente informe corresponde a un estudio comparativo de casos y controles de infección de herida operatoria de artroplastía total y parcial de cadera efectuado en el Servicio de Traumatología del Hospital Clínico San Pablo de Coquimbo, entre enero del 2000 y diciembre de 2004. Los cálculos de costos se realizaron de acuerdo al protocolo de la OPS de 19997.

Los datos fueron obtenidos en forma retrospectiva a partir de los registros de la vigilancia epidemiológica activa efectuada por el Comité de Control y Prevención de Infecciones Intrahospitalarias (CPIIH). La sensibilidad de esta vigilancia en el período de estudio, realizada mediante estudio de prevalencia semestral, fluctuó entre 90 y $100 \%$.

Profilaxis de infección de sitio quirúrgico: todos los pacientes fueron sometidos a la acción de cefazolina sódica, $1 \mathrm{~g}$, administrada una hora antes de la cirugía y dosis sucesivas post operatorias cada ocho horas, por vía endovenosa, durante tres días. Por recomendación del CPIIH del hospital, desde el año 2001 se estandarizó la profilaxis antimicrobiana con una dosis preoperatorio y tres dosis post operatorias en las primeras 24 horas. En todos los casos se utilizó un abordaje de la articulación por vía anterior y se instaló un drenaje aspirativo al vacío que se retiró entre el segundo y tercer día post operatorio.

Selección de los casos. Se consideraron casos los pacientes operados en forma primaria, de artroplastía de cadera, que en el curso del primer año post operatorio presentaron infección superficial (piel y tejidos subcutáneo) o infección profunda (fascia, músculo o implante). Se consideró un seguimiento de un año dado que en presencia de un implante, el período de vigilancia se extiende hasta ese límite ${ }^{8}$. Para establecer el diagnóstico de infección se consideraron las definiciones estandarizadas $\mathrm{y}$ definidas previamente: ${ }^{5,9,10}$, a decir:

- Inflamación y supuración de la herida operatoria

- Descarga líquida de la herida operatoria con cultivo bacteriológico positivo

- Aflojamiento rápido y progresivo, con espacio radiológico lúcido en la interfase hueso cemento

- Dolor constante, con VHS $>30 \mathrm{~mm} / \mathrm{h}$ y PCR sobre $10 \mathrm{mg} / \mathrm{L}$

Un caso fue excluido del estudio porque tenía muchos diagnósticos asociados y no se identificó un control adecuado, pero sí se consideró para los efectos del desenlace.

Selección de los controles. Para la selección de los controles se revisaron todos los pacientes operados en el servicio durante el período de estudio y se eligie- ron aquellos pacientes que no presentaron infección, definiendo dos controles por cada caso. Se restringió la selección a aquellos que tenían la edad del caso \pm tres años, el mismo sexo que el caso índice, mismo diagnóstico principal e igual número de diagnósticos que el caso, \pm uno. Para corroborar la calidad de los controles, un observador independiente confirmó que los controles seleccionados no calificaban como caso, es decir, no tenían infección al momento de realizar el estudio.

Para el cálculo del costo atribuible a la infección, se incluyeron los siguientes parámetros cuantitativos:

- Estadía hospitalaria en el Servicio de Traumatología

- Estadía en Unidad de Cuidados Intensivos

- Número de reintervenciones realizadas en quirófano

- Número de cultivos y antibiogramas registrados en la historia clínica. Las muestras microbiológicas fueron aisladas, identificadas y sometidas a determinación de susceptibilidad a antimicrobianos en el laboratorio de Microbiología del hospital

- Administración de antimicrobianos, expresada en dosis diaria definidas (DDD) y extraídas del registro de indicación médica.

Para valorar los costos de las prestaciones involucradas se consideraron los aranceles de referencia del Fondo Nacional de Salud del Ministerio de Salud vigentes al 30 de junio de 2005, que incluye lo siguientes valores unitarios*:

- Día cama Traumatología \$24.500 (US \$: 47,0)

- Día cama UCI \$ 101.860 (US \$: 194,0)

- Derecho de pabellón e insumos de artroplastía de cadera $\$ 926.500$. (US \$: $1.765,0)$

- Cultivos y antibiograma $\$ 3.410$. (US $\$: 6,5$ )

Como variable de resultados se seleccionaron indicadores económicos de: exceso de días de hospitalización, exceso de uso de antimicrobianos de los pacientes con infección intrahospitalaria, costo del cultivo y antibiograma y costo de las reintervenciones realizadas.

Para evaluar el desenlace se definieron las siguientes condiciones postoperatorias, a partir de la escala de Harris simplificada ${ }^{11}$ :

- Buena: Deambulación sin dolor y sin bastones

- Regular: Deambulación con dolor y/o con bastones

- Mala: Retiro de prótesis sin recambio

- Muerte

Los antecedentes de los pacientes fueron ingresados a la base de datos Epi Info 2000 para su registro y

*Nota: Valor estimado del dólar americano al 15-11-06: US \$1.0= 525,0 pesos 
análisis. El análisis estadístico se realizó a través de la prueba de $t$ de Student para variables no paramétricas, con una significancia de 0,05 , con dos colas.

\section{Resultados}

En el período de estudio, la tasa anual de infección de artroplastía primaria de cadera fue de $2,6 \%$ en el año 2000 (117 pacientes operados); 2,7\% en el año 2001 (111 pacientes operados); 2,1\% en el año 2002 (96 operados); $1,3 \%$ en el 2003 (75 pacientes operados) y $3,6 \%$ en el año 2004 (112 pacientes operados), identificándose 13 casos de infección en los cinco años. En ocho pacientes $(61,5 \%)$ el diagnóstico de infección se estableció en los 30 primeros días post operatorios. Las especies identificadas fueron Staphylococcus aureus en cuatro casos (30,8\%), Staphylococcus coagulasa negativa en cuatro casos, especies aerobias gramnegativas en dos casos; en dos casos no se estableció la etiología.

La media de tiempo de hospitalización en el Servicio de Traumatología fue de 54 días para los casos y 13 días para los controles, siendo esta diferencia estadísticamente significativa $(\mathrm{p}<0,05)$. La media de hospitalización pre-operatoria fue de 9,3 días para los casos y 5,9 días para los controles $(\mathrm{p}=0,44)$. La media de hospitalización en UCI de los casos fue 1,1 días, mientras los controles no tuvieron hospitalización en UCI $(\mathrm{p}=0,33)$; hubo 0,83 reintervenciones en los casos y ninguna en los controles $(\mathrm{p}<0,05)$; por último hubo una media 2,08 cultivos por cada caso y ninguno en los controles $(\mathrm{p}<0,05)$ (Tabla 1$)$.
El total del consumo de antimicrobianos en los pacientes se aprecia en la Tabla 2.

La diferencia de costos por consumo de antimicrobianos entre casos y controles alcanzó a \$1.270.981 (US \$2,421.00) (Tabla 3).

El costo medio de los pacientes infectados, que incluye hospitalización, derecho de pabellón e insumos de artroplastía, reintervenciones, uso de antimicrobianos y cultivos bacteriológicos alcanzó un valor de $\$ 3.241 .800$ (US \$6.174,8). Por su parte, el costo medio de los controles, que evolucionaron sin infección de la artroplastía, alcanzó a \$1.236.244 (US \$2.354,7). El exceso de costo generado por la infección fue de $\$ 2.005 .556$ (US \$3.820,1) (Tabla 4).

La calidad del pareo fue 95\% para edad, $(\mathrm{p}=0,36)$, de $100 \%$ para sexo, diagnóstico principal y procedimiento y de $58 \%$ para número de diagnósticos concomitantes. No hubo pacientes sometidos a tratamiento con corticosteroides. Las enfermedades más frecuen-

Tabla 1. Impacto de la infección de artroplastía de cadera. Hospital Clínico San Pablo de Coquimbo

\begin{tabular}{|c|c|c|c|c|c|}
\hline \multirow[b]{2}{*}{ Variable } & \multicolumn{2}{|c|}{$\begin{array}{l}\text { Casos } \\
(n: 13)\end{array}$} & \multicolumn{2}{|c|}{$\begin{array}{c}\text { Controles } \\
(n: 26)\end{array}$} & \multirow[b]{2}{*}{ T test } \\
\hline & Media & Total & Media & Total & \\
\hline Días de hospitalización en Ortopedia & 53,7 & 644 & 12,5 & 238 & $p<0,05$ \\
\hline Días de hospitalización en UCI & 1,1 & 13 & 0 & 0 & $p=0,33$ \\
\hline Reintervenciones quirúrgicas & 0,83 & 10 & 0 & 0 & $p<0,05$ \\
\hline Cultivos obtenidos & 2,1 & 25 & 0 & 0 & $p<0,05$ \\
\hline
\end{tabular}

Tabla 2. Consumo de antimicrobianos asociado a la infección de artroplastía de cadera. Hospital Clínico San Pablo de Coquimbo

\begin{tabular}{|c|c|c|c|c|c|c|}
\hline Antimicrobiano & Total (g) & $\begin{array}{c}\text { Casos } \\
\text { (n: 13) } \\
\text { Máximo (g) }\end{array}$ & Mínimo (g) & Total (g) & $\begin{array}{l}\text { Controles } \\
\text { (n: 26) } \\
\text { Máximo (g) }\end{array}$ & Mínimo (g) \\
\hline Cloxacilina & 667,5 & 336 & 4 & 0 & 0 & 0 \\
\hline Cefazolina & $299 *$ & 132 & 3 & 195 & 15 & 5 \\
\hline Ciprofloxacina & 158,75 & 78,75 & 10 & 0 & 0 & 0 \\
\hline Vancomicina & 103 & 42 & 7 & 0 & 0 & 0 \\
\hline Amikacina & 80,25 & 33,25 & 8 & 0 & 0 & 0 \\
\hline Ceftazidima & 72 & 72 & 72 & 0 & 0 & 0 \\
\hline Ampicilina/sulbactam & 54,5 & 54,5 & 54,5 & 0 & 0 & 0 \\
\hline Clindamicina & 40,2 & 21,6 & 2,4 & 0 & 0 & 0 \\
\hline Ceftriaxona & 22 & 12 & 4 & 0 & 0 & 0 \\
\hline Rifampicina & 18 & 18 & 18 & 0 & 0 & 0 \\
\hline Gentamicina & 2,86 & 1,9 & 0,8 & 0 & 0 & 0 \\
\hline
\end{tabular}


Tabla 3. Costo global del tratamiento antimicrobiano en infección de artroplastía de cadera. Hospital Clínico San Pablo de Coquimbo

\begin{tabular}{|c|c|c|c|c|c|}
\hline \multirow[t]{2}{*}{ Variable } & \multicolumn{2}{|c|}{ Casos } & \multicolumn{2}{|c|}{ Controles } & \multirow{2}{*}{$\begin{array}{c}\text { Diferencia de costo } \\
\text { US } \$\end{array}$} \\
\hline & $\$$ & US $\$$ & $\$$ & US \$ & \\
\hline \multicolumn{6}{|l|}{ Antimicrobiano } \\
\hline Cloxacilina & 156.862 & 298,7 & 0 & 0 & 298,7 \\
\hline Cefazolina & 101.810 & 193,9 & 66.398 & 122,5 & 71,4 \\
\hline Ciprofloxacina & 6.438 & 12,2 & 0 & 0 & 12,2 \\
\hline Vancomicina & 371.582 & 707,7 & 0 & 0 & 707,7 \\
\hline Amikacina & 93.969 & 178,9 & 0 & 0 & 178,9 \\
\hline Ceftazidima & 89.912 & 171,2 & 0 & 0 & 171,2 \\
\hline Ampicilina / sulbactam & 452.360 & 869,8 & 0 & 0 & 869,8 \\
\hline Clindamicina & 48.635 & 92,6 & 0 & 0 & 92,6 \\
\hline Ceftriaxona & 9.283 & 17,6 & 0 & 0 & 17,6 \\
\hline Rifampicina & 4.248 & 8,0 & 0 & 0 & 8,0 \\
\hline Gentamicina & 2.280 & 4,3 & 0 & 0 & 4,3 \\
\hline Total & 1.337 .379 & $2,547,3$ & 66.398 & 122,5 & $2,424,8$ \\
\hline
\end{tabular}

\section{Tabla 4. Costos económicos promedios ocasionados por la infección artroplastía de cadera. Hospital Clínico San Pablo de Coquimbo}

\begin{tabular}{|c|c|c|c|c|c|}
\hline \multirow[t]{2}{*}{ Variable } & \multicolumn{2}{|c|}{ Casos } & \multicolumn{2}{|c|}{ Controles } & \multirow{2}{*}{$\begin{array}{c}\text { Diferencia de costo } \\
\text { US } \$\end{array}$} \\
\hline & $\$$ & US \$ & $\$$ & US \$ & \\
\hline Hospitalización en Ortopedia & 1.315 .650 & $2,506,0$ & 306.250 & 583,3 & $1.862,7$ \\
\hline Pabellón e insumos & 926.500 & $1,764,7$ & 926.500 & $1,764,7$ & 0 \\
\hline Hospitalización en UCl & 112.046 & 213,4 & 0 & 0 & 213,48 \\
\hline Reintervenciones quirúrgicas & 768.995 & $1,464,7$ & 0 & 0 & $1,464,7$ \\
\hline Cultivos & 7.161 & 13,6 & 0 & 0 & 13,6 \\
\hline Antimicrobianos & 111.448 & 212,2 & 3.494 & 6,6 & 205,6 \\
\hline Total & 3.241 .800 & $6,174,8$ & 1.236 .244 & $2,354,7$ & $3,820,1$ \\
\hline
\end{tabular}

\begin{tabular}{|c|c|c|c|}
\hline Variable & $\begin{array}{l}\text { Casos } \\
(n=12)\end{array}$ & $\begin{array}{l}\text { Controles } \\
(n=19)\end{array}$ & $\begin{array}{c}\text { Porcentaje } \\
\text { de pareo }\end{array}$ \\
\hline Edad & 73 & 70 & $95 \%$ \\
\hline Sexo $M: H$ & $9: 3$ & $13: 6$ & $100 \%$ \\
\hline Diagnóstico principal & $\begin{array}{ll}4 & \text { artrosis } \\
7 & \text { fracturas } \\
1 & \text { tumor }\end{array}$ & $\begin{aligned} 12 & \text { artrosis } \\
7 & \text { fracturas } \\
0 & \text { tumor }\end{aligned}$ & $\begin{array}{r}100 \% \\
100 \% \\
0 \%\end{array}$ \\
\hline $\begin{array}{l}\text { Procedimiento: } \\
\text { Artroplastía total } \\
\text { Artroplastía parcial }\end{array}$ & $\begin{array}{l}7 \\
5\end{array}$ & $\begin{array}{r}14 \\
5\end{array}$ & $100 \%$ \\
\hline Número de diagnósticos & 2,4 & 1,4 & $58 \%$ \\
\hline
\end{tabular}

tes que se presentaron en ambos grupos fueron cardiopatía ateroesclerótica y enfermedad pulmonar obstructiva crónica.

El desenlace de la artroplastía de cadera en los 13 pacientes infectados fue el siguiente:

- Buena función, sin dolor, sin claudicación, sin bastones, sube escalas y se coloca calcetines (función normal): cuatro pacientes $(30,8 \%)$

- Regular con dolor y/o bastones: cinco pacientes $(38,5 \%)$

- Mala con nueva cirugía para retiro de prótesis y sin recambio (postrado): un paciente $(7,7 \%)$

- Muerte (sepsis): dos pacientes $(15,4 \%)$

- Perdido de seguimiento: un paciente $(7,7 \%)$. 


\section{Discusión}

El riesgo de infección de la artroplastía de cadera se ha asociado a factores tan diversos como el volumen de cirugía anual, la ausencia de personal a cargo de la vigilancia epidemiológica ${ }^{12}$, uso de heparina de bajo peso molecular para la prevención de la trombosis venosa ${ }^{13}$, uso de corticosteroides intra-articulares previos $^{14}$, edad avanzada de los pacientes, sexo femenino, riesgo anestésico: ASA score II y III, índice de masa corporal mayor de 25 , estadía pre-operatoria mayor de cuatro días ${ }^{6}$, presencia de artritis reumatoide y/o diabetes mellitus, calidad de la técnica quirúrgica, duración del tiempo operatorio ${ }^{15}$, uso de hemotransfusiones alogénicas ${ }^{16} \mathrm{y}$ uso de drenajes en la herida operatoria. En este estudio, estos factores no fueron evaluados directamente; sin embargo, al evaluar la calidad de pareo se aprecia que en los casos predominaron las mujeres y la media de edad era de 73 años, lo que es consistente con los factores de riesgo conocidos de sexo femenino y edad avanzada ( $>65$ años).

El abordaje quirúrgico posterior se ha considerado un factor de riesgo para infección; sin embargo, un meta-análisis reciente no ha confirmado este riesgo ${ }^{17}$. No obstante, en nuestro hospital la vía anterior es el procedimiento para realizar la operación.

La calidad de los microorganismos identificados en la infección de la artroplastía y la frecuencia de ocurrencia de estos es similar a la descrita por Mayhall en una serie recopilada de más de 2.000 pacientes infectados ${ }^{18}$.

Si los pacientes presentan una evolución sin incidentes, la hospitalización representa el 50\% de los costos hospitalarios y el implante da cuenta de alrededor de $25 \%$ de los costos totales, dependiendo del tipo de prótesis ${ }^{19}$. La media de costo de la artroplastía primaria es de US \$6,080 en Canadá y de US \$12,846 en E.U.A., con una media de hospitalización de 7 y 4 días, respectivamente ${ }^{20}$. En nuestro hospital, dependiente de la red estatal de salud, ese costo en pacientes sin complicaciones es menor (US \$ 3,726 menos que en Canadá y US \$10,492 menos que en E.U.A.) debido a los mecanismos de subsidio que el Estado de Chile genera al fijar los costos de hospitalización y otros costos asociados, bajo los valores comerciales.

En la literatura médica existen pocos informes que precisen los costos que genera la infección intrahospitalaria de la artroplastía de cadera. Un estudio recien- te de $\mathrm{P}$. Brenner ${ }^{21}$ señala que los controles tienen un promedio de estada de 16 días y los casos de infección presentan una media de estada de 66 días, valores similares a nuestros resultados de 13 y 54 días, respectivamente, pero en aquel no se definen datos de indicadores monetarios.

En resumen, todos los factores medidos en nuestro estudio contribuyeron a encarecer los costos de los pacientes infectados, que deben ser financiados por el hospital y por el paciente, con un enorme impacto físico, psicológico, social, financiero y legal para todos los actores involucrados en esta devastación, que comprometen la supervivencia de la extremidad y, a veces, la vida misma del paciente ${ }^{22}$. La letalidad de nuestra serie $(15,4 \%)$ refleja la gravedad de la infección de la herida en artroplastía de cadera. Todos los esfuerzos destinados a evitar la infección se justifican ampliamente a la luz de estos resultados, en especial, a través de la adherencia a las buenas prácticas clínicas de todo el personal involucrado en esta cirugía y a través de una vigilancia epidemiológica estricta que permita identificar los factores de riesgo más probables asociados a cada caso, así como generar programas de intervención destinados a neutralizar esos factores de riesgo.

\section{Resumen}

Estudio de casos y controles efectuado para evaluar el costo y desenlace de la infección del sitio quirúrgico en artroplastía primaria de cadera en cinco años. La media de hospitalización en el Servicio de Traumatología fue de 54 días para los casos y 13 días para los controles $(p<0,05)$. La media de hospitalización en UCI de los casos fue 1,1 días; hubo 0,83 reintervenciones en los casos y una media de 2,08 cultivos por cada caso. Los controles no tenían hospitalización en UCI, reintervenciones, ni cultivos. El costo promedio de los pacientes infectados fue $\$ 3.241 .800$ (US $\$ 6.174,8)$. El costo promedio de los controles, alcanzó a \$1.236.244 (US \$ 2.354,7). El exceso promedio de costo por la infección fue de $\$ 2.005 .556$ (US $\$$ $3.820,1)$. El desenlace fue el siguiente: función normal $30,8 \%$; secuelas medianas y graves $46,2 \%$; letalidad $15,4 \%$. Los factores medidos contribuyeron a encarecer los costos de los casos y produjeron un desenlace insatisfactorio para dos tercios de los pacientes. 


\section{Referencias}

1.- Brady O H, Masri B A, Garbuz D S, Duncan C P. Rheumatology 10. Joint replacement of the hip and knee: when to refer and what to expect. Can Med Assoc J 2000; 163, 14: 1285-91.

2.- Charnley J. Arthroplasty of the hip: a new operation. Lancet 1961; 1: 1129.

3.- Aamodt A, Nordsletten L, Havelin LI. Documentation of hip prostheses used in Norway: a critical review of the literature from 1996-2000. Acta Orthop Scand 2004; 75: $663-76$.

4.- Hunter D, Robertson D. Total hip replacement: need far exceeds supply. Can Med Assoc J 2001; 165: 395.

5.- Fica A, Llanos C, Luzoro A, De la Barrera C, Miranda G. Infecciones en prótesis articulares. Rev Chil Infect 2000; 17: 115-21.

6.- Ridgeway S, Charlet A, Kafatos G, Pearson A, Coello R. Infection of the surgical site alters arthroplasty of the hip. J Bone Joint Surg 2005; 87: 844-50.

7.- Organización Panamericana de la Salud 1999. Protocolo para el cálculo de costos de la infección intrahospitalaria. http://www. paho.org/Spanish/AD/DPC/CD/protocolo. pdf

8.- Wong E. Surgical site infections. En Mayhall CG: Hospital Epidemiology \& Infection Control. Third Ed Lippincott: 288. (Falta año, editorial y ciudad de publicación)
9.- Otaíza F, Brenner P. Ministerio de Salud de Chile 1998: Sistema de vigilancia de las infecciones intrahospitalarias.

10.- Bergallo C. Infección de prótesis de cadera: Paradigma de las infecciones de prótesis articulares. Rev Chil Infect 2000; 17: 87-91.

11.- Reyna G, Harb E. Comparación clínico radiológica del fresado y no fresado acetabular en la hemiartroplastía de cadera. Acta Ortop Mex 2004; 18: 96-9.

12.- Geubbels E, Willie J, Nagelkerke N, Vandenbroucke-Grauls C M, Groebbee de Boer S A. Hospital related determinants for surgical site infection following hip arthroplasty. Infect Contr Hosp Epidemiol 2005; 26: 435-41.

13. - Sánchez Ballester J, Smith M, Hasan K, Kershaw S, Ellsworth E C, Jacobs L. Wound infection in the management of a hip fracture: a comparison between low molecular weight heparin and mechanical prophylaxis. Acta Orthop Belg 2005; 71: 55-9.

14.- Kaspar S, de Beer J. Infection in hip arthroplasty alters previous injection of steroids. J Bone Joint Surg Br 2005; 87: 454-7.

15.- Otaíza F, Brenner P. Infecciones en cirugía de artroplastía de cadera: resultados del sistema de vigilancia epidemiológica de las infecciones intrahospitalarias. 1996-1999. Rev Chil Infect 2000; 17: 109-14.
16.- Innerfofer $\mathrm{P}$, Klingler $\mathrm{A}$, Klimmer $\mathrm{C}$, Fries D, Nussbaumer W. Risk for postoperative infection alters transfusion of white blood cell filtered allogenic or autologous blood components in orthopedic patients undergoing primary arthroplasty. Transfusion 2005; 45: 103-10.

17.- Jolles B M, Bogoch E R. Surgical approach for total hip arthroplasty: direct lateral or posterior? J Reumathol 2004; 31: 1790-6.

18.- Lew D, Pittet D, Waldvogel F. Infections that complicate the insertion of prosthetic devices. En Mayhall CG: Hospital Epidemiology \& Infection Control. Third Ed Lippincott, pp: 1186.

19.- Scheerlinck T, Duquet W, Casteleyn PP. Socioeconomic aspects of total hip artrhoplasty. Acta Orthop Belg 2004; 70: 525-33.

20.- Antoniou J, Martineau P A, Filion K B, Haider S, Zukor D J, Huk L, et al. In hospital costs of total hip arthoroplasty in Canada and the United States. J Bone Joint Surg Am 2004; 86-A: 2435-9.

21.- Brenner P, Nercelles P, Polhenz M. Costo de las infecciones intrahospitalarias en hospitales chilenos de alta y mediana complejidad. Rev Chil Infect 2003; 20: 285-90.

22.- Kaltsas DS: Infection after total hip arthroplasty. Ann R Coll Surg England 2004; 86: 267-71. 